\title{
Only one step from tailored oncological therapies
}

\author{
Paweł Krawczyk
}

The personalization of oncological therapy is becoming a reality thanks to the development of molecular biology techniques (e.g. next generation sequencing, liquid biopsy) and the discovery of new molecularly targeted therapies and immunotherapeutics. Genetic laboratories and clinical centers, which form international networks as well as global, early phase clinical trials make it possible to match treatments to a particular patient. The benefit of such a procedure is currently difficult to estimate, but it seems that another path for the development of oncology is not possible.

NOWOTWORY J Oncol 2018; 68, 1: 42-45

Key words: oncology, molecularly targeted therapies, immunotherapies, gene sequencing, liquid biopsy

\section{Introduction}

Tailored oncological treatment based on molecularly targeted therapies and immunotherapies play increasing role in the multidisciplinary approach to cancer patient care. Treatment personalization extends the scope of therapy and extends the survival of patients. An example of lung cancer treatment personalization is our 57-year-old male patient with advanced lung adenocarcinoma and activating EGFR gene mutations, as well as PD-L1 expression. As the methods of treatment, we used segmentectomy (diagnostic manner), hemipelvectomy of metastases in the right hip joint with postsurgical radiotherapy, surgical cytoreduction, and six lines of systemic treatment which were based on standard cytostatics and novel personalized agents. The presence of the coexistence of rare mutations in exon 18 of the EGFR gene (G719X and G709X) in tumor cells allowed the use of the first-generation (erlotinib) of tyrosine kinase inhibitor (TKI) of EGFR (epidermal growth factor receptor). The appearance of the Thr790Met mutation in exon 20 of the EGFR gene, in circulating tumor DNA (ct-DNA) from peripheral blood, during the progression to erlotinib allowed the use of the third generation of EGFR-TKI (osimertinib). The presence of PD-L1 expression on tumor cells justified the attempt to apply immunotherapy (nivolumab - anti-PD-1 antibody). The patient also received a tyrosine kinase inhibitor of VEGFR, FGFR and PDGFR (nintedanib) in combination with chemotherapy. As a result, we have achieved long-term overall survival with long progression-free periods during individual therapy [1].

Of course, such a therapeutic approach could be regarded as unattainable for larger groups of patients. Personalized therapies are available only in developed countries with access to modern genetic methods of cancer diagnosis and to clinical trials with novel drugs. Today, personalized therapies could be used in a small percentage of patients with a genetic predisposition to the treatment. Moreover, cancer cells can develop resistance to even the best molecularly targeted therapies and immunotherapies. Tumors are also molecularly heterogeneous, so it is difficult to find for our patients appropriate and permanently effective personalized therapy. However, the development of new molecular methods (especially next generation sequencing and liquid biopsy) and the discovery of new drugs investigated in clinical trials raise the hope that precise oncology has arrived.

\section{Next generation sequencing and liquid biopsy in qualification to personalized treatment in cancer patients}

Next generation sequencing (NGS) is a new DNA sequencing technology which has revolutionized genomic research. Using NGS, the whole genome of cancer cells

Department of Pneumonology, Oncology and Allergology, Medical University of Lublin, Poland 
(whole genome sequencing,WGS) can be sequenced within a single day, and the price of the examination has dropped so much that WGS could be available to even individual patients. In contrast, the previous Sanger direct technology required over a decade in order to map the human genome. NGS is increasingly translated into routine clinical practice, especially in oncology and in pediatrics. NGS could be used to sequence whole genome or specific genome areas, including coding sequences (whole exome sequencing, WES) and individual genes (e.g. oncogenes with "druggable" mutations). There are a number of different NGS platforms using different sequencing technologies. In a very simplistic way, all NGS technologies use three stages [2,3]:

1. Library preparation with DNA fragmentation and in vitro adaptor ligation.

2. Clonal amplification using an emulsion or bridge PCR (polymerase chain reaction).

3. Cyclic sequencing of millions of small fragments of DNA in parallel using pyrosequencing, sequencing by ligation or sequencing by synthesis.

WES or sequencing of selected genes are most commonly used in the personalization of oncological treatment. The sequencing of frequently mutated genes in tumor cells in particular malignancies, is the most meaningful, cost-effective and rapid method to qualify to molecularly targeted drugs. These mutations should cause changes in proteins involved in intracellular pathways which could be blocked by molecularly targeted drugs. WES may be a useful method to evaluate the number of mutations in tumors (tumor mutation burden) in qualification to immunotherapy (a large number of mutations results in the increased number of tumor-specific antigens and increased efficacy of immunotherapy) $[2,3]$.

Several diagnostic companies propose the sequencing of selected genes to qualify individual patients for molecularly targeted therapies (e.g. FoundationOne, OncoDNA, ONCOMPASS, Caris, Guardant 360). NGS is not only offered to detect gene mutations (indels, substitutions, single nucloetide transcriptional variants (SNVs), but also for gene copy number variations (CNV) and chromosome translocation (rearrangement) analysis or epigenetic analysis (promoter genes methylation). In offers, NGS analysis is supplemented by simpler genetic methods, e.g. immunohistochemistry (IHC) for assessment of protein expression, fluorescence in situ hybridization (FISH) for chromosomal abnormality analysis, and real-time PCR technique for single mutation analysis. The patients' report should consist not only of the result of genetic tests, but also of the the analysis of the availability of personalized treatment (chemotherapy, molecularly targeted therapies, immunotherapy). Such a report takes into account therapies registered for treatment of particular cancers, therapies registered for other indications (potentially useable due to the occurrence of genetic alterations), and drugs in clinical trials (often available in distant locations) [2,3].

The possibility of genetic diagnostics and qualification for molecularly targeted therapies and immunotherapy has considerably expanded through the introduction of analysis of genetic materials from peripheral blood (liquid biopsy), with highly sensitive molecular methods (e.g. some NGS techniques, droplet digital PCR and BEAMing PCR). Circulating tumor DNA (ctDNA) is now being extensively studied, as it is a noninvasive "real-time" biomarker that can provide diagnostic and prognostic information before, during treatment and at progression. These include DNA mutations, epigenetic alterations and other forms of tumor-specific abnormalities such as microsatellite instability (MSI) and loss of heterozygosity ( $\mathrm{LOH}$ ). The major challenge of genetic analysis of material from liquid biopsy is assay sensitivity. Frequent EGFR gene mutations (deletions in exon 19 and Leu858Arg substitution in exon 21) are detected in liquid biopsies of non-small cell lung cancer (NSCLC) patients with a sensitivity of $60-75 \%$. Therefore, the tumor tissue or cells are the most important material for examination of these mutations during qualification of NSCLC patients to EGFR-TKI therapy. However, the use of liquid biopsy for detection of EGFR mutations, responsible for EGFR-TKI resistance during disease progression (e.g. Thr790Met substitution in exon 20), extends the ability of patient qualification to third generation EGFR-TKI (osimertinib). Moreover, analysis of genetic materials from liquid biopsy allows determination of the moment of molecular progression (the appearance of genetic material of cancer cells in peripheral blood). In addition, examination of genetic mutations in ctDNA allows the selection of appropriate molecularly targeted compounds. A good example is the treatment of NSCLC patients with $A L K$ gene rearrangement using various ALK inhibitors (crizotinib, ceritinib, alectinib, lorlatinib). Recently identified $A L K$ gene mutations are responsible for differences in the sensitivity of ALK inhibitors. Therefore, identification of the appearance of $A L K$ mutations during ALK inhibitor treatment allows qualification to appropriate next generation drugs and selection of the appropriate treatment sequence for these drugs [4-6].

\section{The concept of an oncology center network with access to molecular diagnosis and clinical trials with new molecularly targeted therapies}

The National Comprehensive Cancer Network developed clinical practice guidelines in oncology for non-small-cell lung cancer diagnosis and treatment. These guidelines include recommendations to perform tests for EGFR mutation and $A L K$ rearrangement detection, and also examination of BRAF V600E mutation, MET amplification, MET exon 14 skipping mutation, RET rearrangements and HER2 mu- 
tations. Targeted agents studied in advanced clinical trials are available for patients with these genetic abnormalities. Genetic abnormalities in more than 10 genes in tumor cells have become "druggable" in lung cancer patients [6]. The Lung Cancer Mutation Consortium published data about the effectiveness of molecularly targeted therapies in molecularly predisposed lung cancer patients. The median overall survival (OS) of patients with different "druggable" mutations, who received matched molecularly targeted therapies, was significantly longer than the median OS of patients without genetic abnormalities or patients with genetic alterations who could not be treated with molecularly targeted therapies. The organization of such a clinical trial and the availability of a variety of molecularly targeted therapies and genetic tests, were possible thanks to the participation of many American clinical centers. Similar multi-center diagnostic and clinical networks operate in EU countries (e.g. Cancer Research UK). Such cooperation enabled the organization of several clinical trials in which treatment was personalized based on the molecular profile of cancer cells, in patients with different types of neoplasms [7].

The IMPACT 1 study enrolled 3536 patients with 15 different advanced malignancies. In 2089 patients (59\%), at least one "druggable" genetic abnormality was detected, and more than 3 such abnormalities were diagnosed in 359 (10.2\%). Patients with melanoma, as well as with breast, endometrium, colorectal and ovarian cancer, presented genetic abnormalities in tumor cells most often. Response to treatment and disease control were reported in $21 \%$ and $42 \%$ of the patients treated with a therapy based on the molecular profile of cancer cells. In comparison, in patients without feasibility of tailored therapy, the response rate to chemotherapy was $6 \%$, and disease control was observed in $19 \%$ of cases. The use of tailored therapy enabled prolongation of progression free survival from 2.4 to 4.1 months and overall survival from 8.2 to 10.2 months. Based on these findings, the IMPACT 2 study was started in patients with advanced solid tumors [8].

It is worth mentioning that other clinical trials have been conducted on a similar basis, and their results are expected in the coming months. The multicenter SWOG S1400 phase II clinical trial enrolled patients with advanced squamous cell lung cancer. PIK3CA mutations, CCND1 amplification, CDKN2 deletion, RB mutations, FGFR mutations, rearrangement and amplification, as well as MET expression, were examined in all SCC patients. Based on the detected genetic abnormalities, patients could be treated with inhibitors of PIK3CA, CDK 4 or 6, FGFR and HGF in combination with chemotherapy or erlotinib [9]. The BATTLE-2 study was dedicated to advanced NSCLC patients. Genetic abnormalities detected in cancer cells determined the use of inhibitors of EGFR (erlotinib), RAF (sorafenib), MEK (selumetinib) or AKT (MK 2206) [10].
Selection of individualized treatments based on genetic analysis of paired tumor and normal tissue was performed in the global WINTHER study. In 200 patients biopsies of metastatic lesions and matched normal tissue were executed in order to obtain material for whole genome sequencing. Patients with molecular predictive factors received matched molecular targeted therapies including drugs in the opened 1st phase clinical trials. In patients without such predictive factors, oncologists used therapeutic choice based on predictive drug efficacy scoring [11].

The SHIVA clinical trials enrolled patients with recurrent and refractory to standard treatment solid tumors. Molecular profiling using the NGS technique is performed in tumor biopsy. After centralized bioinformatics analysis of the genetic results, the molecular biology board makes the treatment decision. Eligible patients are randomized to molecularly targeted therapies or to treatment at the physician's choice. Treatment cross over is possible at the time of disease progression $[12,13]$.

The National Cancer Institute - MATCH Clinical Trial explores the possibilities of the introduction to routine practice of novel treatment methods based on molecular profiles of patients' tumors. About 6000 patients with solid tumors (including rare tumors, lymphomas and myeloma) that no longer respond to standard treatment are screened with a tumor biopsy. Changes in 143 genes are diagnosed using the NGS technique. If a patient's tumors have genetic abnormalities that match the one targeted by a drug used in clinical trial, the patient are eligible to join the treatment portion of $\mathrm{NCl}-\mathrm{MATCH} .30$ different therapies that target 30 different genetic abnormalities are available for cancer patients taking part in this clinical trial $[3,14]$.

\section{Conclusion}

Tremendous progress in molecular biology and the development of new molecularly targeted drugs allow treatment personalization for very narrow, genetically selected groups of cancer patients. The nature of clinical trials is evolving. In the previous decade, large clinical trials of the 3rd phase have been dominant, enrolling hundreds and even thousands of patients. Currently, early phase clinical trials enroll only individual patients with a genetic predisposition to an individual treatment method. Therefore, the effectiveness of the investigated drugs is discussed on the basis of individual case reports. Patients actively search for such clinical trials, traveling around the world for treatment. Often, such an attitude prolongs their life just enough to make even one of their dreams come true.

Conflict of interest: none declared

Prof. Pawel Krawczyk MD, PhD

Medical University of Lublin

Department of Pneumonology, Oncology and Allergology 


\section{ul. Jaczewskiego 8}

20-954 Lublin, Poland

e-mail:krapa@poczta.onet.pl

Received \& Accepted: 4 Jul 2017

Based on the presentation at the V Annual Conference of the Nowotwory Journal of Oncology, 'Oncological Debates', held in Warszawa, 7-8th April 2017

\section{References}

1. Novello S, Barlesi F, Califano R et al. Metastatic non-small-cell lung cancer: ESMO Clinical Practice Guidelines for diagnosis, treatment and follow-up. Ann Oncol 2016; 27 (suppl 5): v1-v27.

2. Gagan J, Van Allen EM. Next-generation sequencing to guide cancer therapy. Genome Med 2015; 7: 80.

3. Lih CJ, Harrington RD, Sims DJ et al. Analytical validation of the next-generation sequencing assay for a nationwide signal-finding clinical trial: Molecular Analysis for Therapy Choice clinical trial. J Mol Diagn 2017; 19: 313-327.

4. Diaz LA, Bardelli A. Liquid biopsies: genotyping circulating tumor DNA. J Clin Oncol 2014; 32: 579-586.

5. Mack P. Emerging role of liquid biopsies in NSCLC. 17th World Conference on Lung Cancer. Abstract. SC21.05.

6. Tsao AS, Scagliotti GV, Bunn PA Jr et al. Scientific advances in lung cancer 2015. J Thorac Oncol 2016; 11: 613-638.
7. Kris MG, Johnson BE, Berry LD et al. Using multiplexed assays of oncogenic drivers in lung cancers to select targeted drugs. JAMA 2014; 311: 1998-2006

8. Tsimberidou AM, Wen S, Hong DS et al. Personalized medicine for patients with advanced cancer in the phase I program at MD Anderson: validation and landmark analyses. Clin Cancer Res 2014; 20: 4827-4836.

9. Herbst RS, Gandara DR, Hirsch FR et al. Lung Master Protocol (Lung$\mathrm{MAP}$ ) - a biomarker-driven protocol for accelerating development of therapies for squamous cell lung cancer: SWOG S1400. Clin Cancer Res 2015; 21: 1514-1524.

10. Papadimitrakopoulou V, Lee JJ, Wistuba II et al. The BATTLE-2 Study: A Biomarker-Integrated Targeted Therapy Study in Previously Treated Patients With Advanced Non-Small-Cell Lung Cancer. Clin Oncol 2016, 34: 3638-3647.

11. Rodon J, Soria JC, Berger Ret al. Challenges in initiating and conducting personalized cancer therapy trials: perspectives from WINTHER a Worldwide Innovative Network (WIN) Consortium trial. Ann Oncol 2015; $26: 1791-1798$

12. Belin L, Kamal M, Mauborgne $\mathrm{C}$ et al. Randomized phase II trial comparing molecularly targeted therapy based on tumor molecular profiling versus conventional therapy in patients with refractory cancer: cross-over analysis from the SHIVA trial. Ann Oncol 2017; 28: 590-596.

13. Desportes $E$, Wagner $M$, Kamal $M$ et al. Prognostic factors of successful on-purpose tumor biopsies in metastatic cancer patients included in the SHIVA prospective clinical trial. Oncotarget 2017; 8: 1760-1773.

14. Mullard A. NCl-MATCH trial pushes cancer umbrella trial paradigm. Nat Rev Drug Discov 2015; 14: 513-515. 